Therefore, the summed power over all the amplifiers on the feedback stage is

$$
\begin{aligned}
P_{f}=\sum_{l=k+1}^{n-1} P_{l}=\sum_{l=k+1}^{n-1} R_{m l} & \left(\sum_{j=k+1}^{n-1} R_{m j} y_{l j}\left|Y_{T}\right|^{2}\right. \\
& \left.+\sum_{i=1}^{k} K_{i} y_{l i} \bar{Y}_{T}\right)\left|E_{1}\right|^{2} .
\end{aligned}
$$

From (A-3) and (A-6), the total power is given by the following:

$$
\begin{aligned}
P_{\mathrm{a} l 1}= & P_{\mathrm{in}}+P_{f} \\
= & {\left[\sum_{i=1}^{k} \sum_{j=1}^{k} K_{i} K_{j} y_{i j}+\sum_{l=k+1}^{n-1} \sum_{j=k+1}^{n-1} R_{m l} R_{m j} y_{l j}\left|Y_{T}\right|^{2}\right.} \\
& \left.+2 \sum_{l=k+1}^{n-1} \sum_{i=1}^{k} R_{m l} K_{i} y_{i l} \operatorname{Re}\left(Y_{T}\right)\right]\left|E_{1}\right|^{2}
\end{aligned}
$$

\section{ACKNOWLEDGMent}

The author would like to thank Prof. T. Yanagisawa of Tokyo Institute of Technology. Many thanks are also due to Prof. M. Kawakami and the author's collegues for their useful discussions.

\section{REFERENCES}

[1] R. P. Sallen and E. L. Key, "A practical method of designing RC active filters," IRE Trans. Circuit Theory, vol. CT-2, pp. 74-85, Mar. 1955.

[2] N. Fujii, "Active RC synthesis of transfer functions with adjustable .coefficients," Proc. Inst. Elec. Eng., vol. 117, no. 11, p. $2109,1970$.

[3] T. Fjällbrant, "Canonical realization of non-minimum phase transfer functions by means of active and non-reciprocal elements," Ericsson Tech., vol. 27, no. 2, p. 214, 1967.

[4] E.S. Kuh, "Transfer function synthesis of active RC networks," IRE Trans. Circuit Theory (Special Supplement), vol. CT-7, pp. 3-7, Aug. 1960.

[5] L. Weinberg, Network Analysis and Synthesis. New York: McGraw-Hill, 1962.

[6] E. A. Guillemin, Synthesis of Passive Networks. New York: Wiley, 1957.

\title{
A Universal Low-Q Active-Filter Building Block Suitable for Hybrid-Integrated Circuit Implementation
}

\author{
GEORGE S. MOSCHYTZ
}

\begin{abstract}
A highly versatile second-order active-filter building block for low- $Q$ applications $(Q \leq 25)$ is described. The versatility is obtained by combining features inherent in the Sallen-Key circuit topology and those pertaining to hybrid-integrated circuits (HIC's). A high degree of circuit stability is obtained by designing individual network functions to depend primarily on the characteristics of stable thin-film resistors and capacitors.

Frequency- and $Q$-sensitivity are examined in detail. Methods of minimizing $Q$-variation with respect to the active and passive elements are discussed. Lower bounds on the $Q$-variation obtainable with the hybrid-integrated filter building blocks are given.

All possible second-order network functions, including the allpass function, can be obtained with the building block. The resulting. universality and standardization permit the complete design of active filters consisting of cascaded building blocks by computer.
\end{abstract}

\section{INTRODUCTION}

$\bigwedge$ LARGE NUMBER of filtering functions used in communications systems can be realized with relatively low pole- $Q$ 's. Typically, these may

Manuscript received March 7, 1972; revised June 10, 1972.

The author was with Bell Telephone Laboratories, Inc., Holmdel, N. J. 07733. He is currently on a leave of absence at the Swiss Federal Institute of Technology, Zurich, Switzerland. range from less than 1 to 25 . Pole- $Q$ 's of this magnitude can be built in active $R C$ form with good stability using only one operational amplifier. Since the cost of supplying power to active filters is appreciable and the heat generated by the dissipated power not insignificant, there is much to be gained by using single-amplifier active filters wherever the pole- $Q$ 's are low enough to permit this.

A single-amplifier filtering method for low- $Q$ filter applications is described here. Whereas the method is based on the Sallen-Key circuit scheme [1], it expands on this scheme in two ways.

Firstly, it considers Sallen-Key circuits from the point of view of hybrid-integrated circuit (HIC) implementation. Seen from this vantage point, the apparent disadvantage of this positive feedback filtering scheme, namely its high $Q$-sensitivity to gain and component variations, can be eliminated. Indeed, from this point of view it seems superior to many negative feedback singleamplifier filtering methods, all of which, like SallenKey's, are restricted to low- $Q$ applications if reasonable $Q$-stability is to be guaranteed. One of the reasons for 
this superiority is the low gain required by the SallenKey circuits, which are of the controlled-voltage source type. When used in the unity or close-to-unity gain mode, such circuits can operate satisfactorily up to higher frequencies than many single-amplifier schemes requiring high or "infinite" gain. Another feature that makes the Sallen-Key method particularly suitable for HIC implementation is the fact that it requires a much smaller spread of component values than most other single-amplifier methods. This is important because thin-film resistors and capacitors can be expected to track closely only if they do not differ greatly in value (i.e., line width and surface area); it is precisely the excellent tracking capability of HIC resistors and capacitors that makes these components so attractive to the circuit designer and provides highly stable circuits.

Secondly, in accordance with the demands of integrated circuit (IC) processing, a building-block approach to filter design has been developed using the Sallen-Key circuit topology as a starting point. The resulting singleamplifier low- $Q$ filter building block demonstrates extraordinary versatility, since it is capable of providing every possible second-order minimum and nonminimum phase network function, using the same basic circuit topology for each. The building block is directly compatible with HIC technology and, as numerous practical examples have demonstrated, the electrical performance corresponds very accurately to theoretical predictions.

One of the advantages of these low- $Q$ building blocks is that any section can be readily extended to high- $Q$ operation by simply adding a frequency-emphasizing network (FEN) in cascade with it [2]. The added power dissipation of the two-amplifier FEN building block is thereby used only when warranted to augment $Q$. The result is a very flexible and economical filter buildingblock system that has been used with excellent results in various exploratory data transmission terminals.

Another advantage of these building blocks results from the fact that transmission zeros for elliptic-filtcr functions are generated by passive $R C$ networks, namely by a twin- $T$, rather than by a differential-input operational amplifier. Thus the transmission zeros depend on passive $R C$ components only and the resulting filter sections belong to the class of networks whose transmission sensitivity to the most variable element - namely gain -depends only on the pole locations [3]. The important consequence is that pole-zero pairing of $n$ th-order network functions into second-order functions can be carried out exclusively with a view to optimizing signal level and minimizing distortion [4.], without regard for sensitivity minimization. This freedom is not shared by networks in which zeros are generated by the commonmode rejection of differential-input operational amplifiers.

By realizing the transmission zeros of the filter building block with a twin- $T$ (independent of amplifier characteristics), the zeros and poles can be tuned virtually

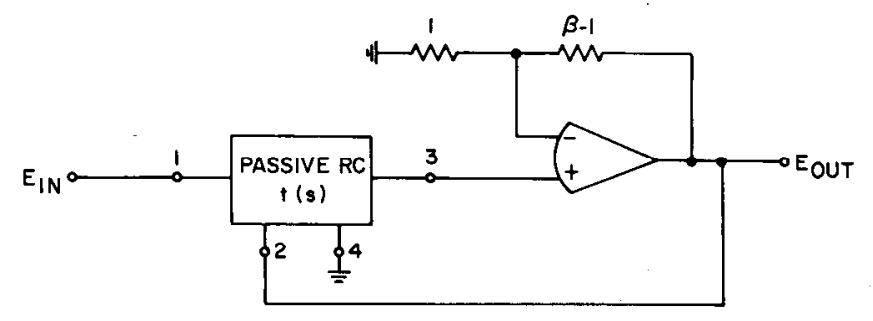

(a)

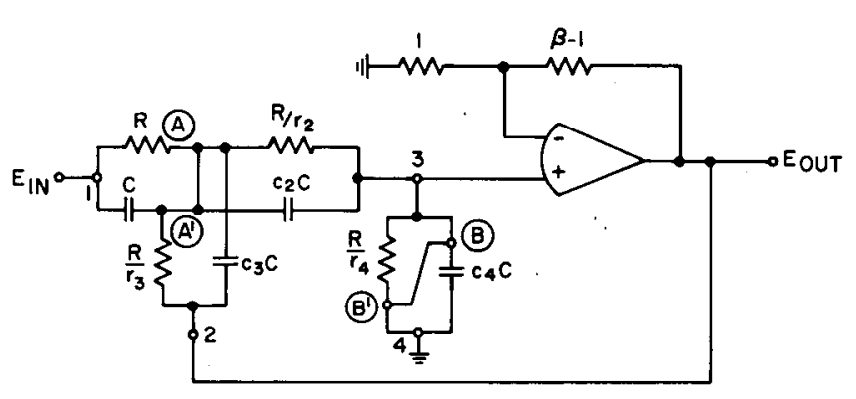

(b)

Fig. 1. Sallen-Key circuit. (a) Basic topology.

(b) Circuit for general-purpose SABB.

independent of one another. Features like this that actually make the twin- $T$ especially suitable for tuning when in HIC form are worth mentioning here, since they conflict somewhat with the experiences recalled by anyone who has had the frustrating assignment of tuning, and maintaining, a null with a discrete twin-T network.

This last point brings up some observations which, although seemingly obvious, are of ten overlooked whenever various active filter schemes are compared with one another. Whereas the twin-T network has proven to be an excellent building block in HIC oscillator [5], [6] and filter design [7], it cannot be recommended with the same enthusiasm for discrete linear networks. The main reason for this is that tunable discrete components, with the same stability as those of HIC's, are either unavailable or too costly. This demonstrates once again how closely related a given approach to circuit design is, and should be, to the technology used for its optimum implementation. Proven circuit designs of an established technology should not be forced onto new emerging technologies, but should be appropriately modified and adapted to them to fully benefit from the characteristics they afford. Thus, in comparing active filter methods, it is not possible to derive much valid meaning from a comparison of methods per se, without considering these methods within the context of the technological implementation for which they are intended. There is no question that the filter building blocks described here will perform satisfactorily only when realized in the HIC technology for which they were intended, and that any one of numerous other methods may have been selected for discrete component implementation. In the light of the observations above, however, this is exactly as it should be. 
TABLE I

SF.COND-ORDF.R S\&K NFTWORKS

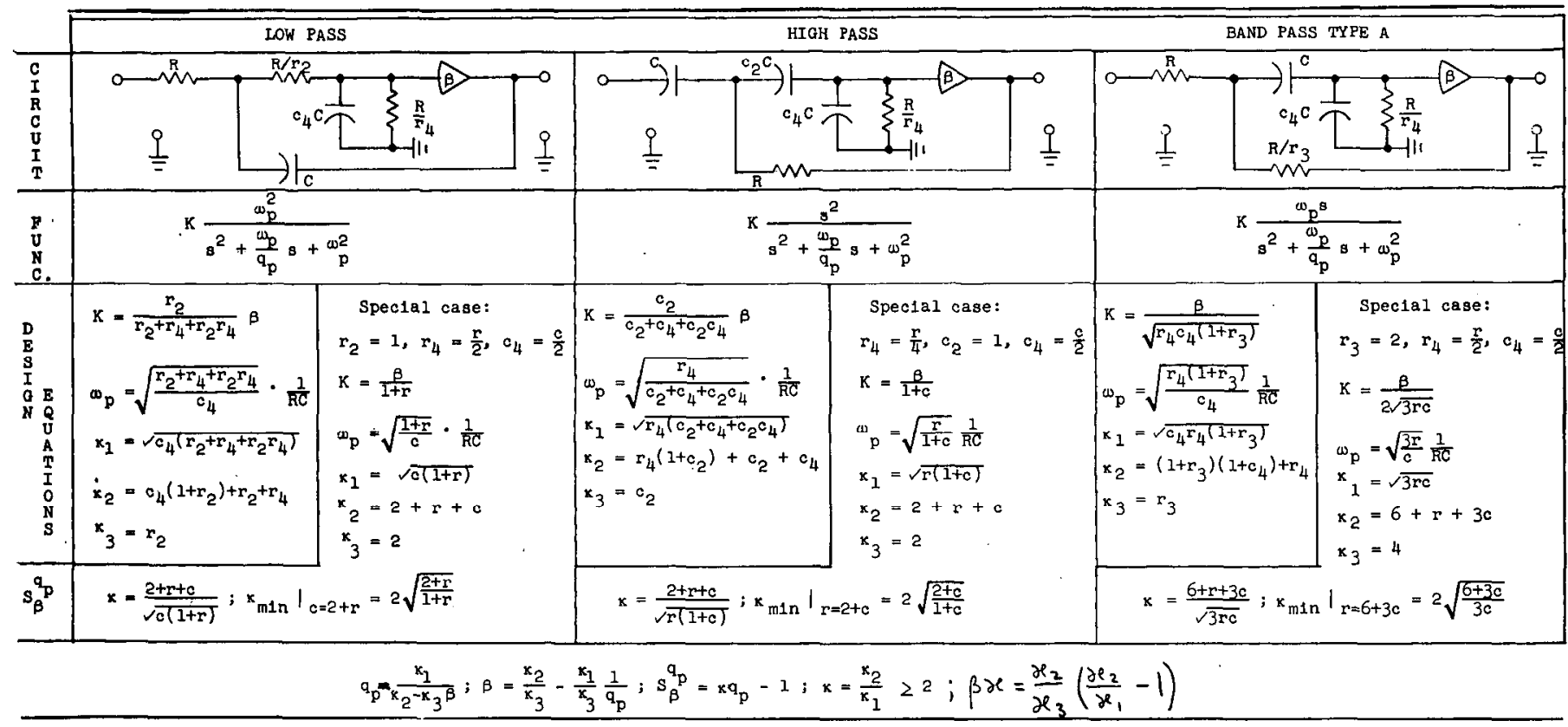

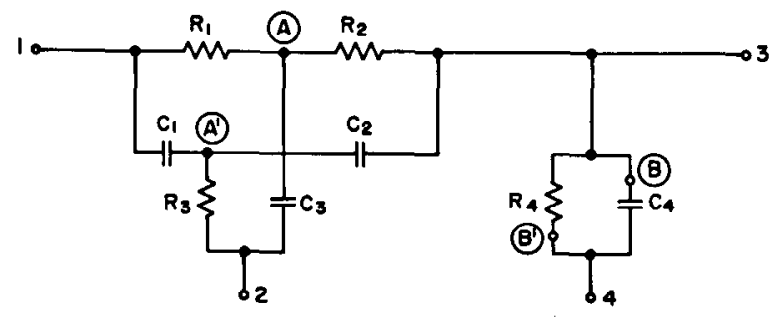

Fig. 2. $R C$ feedback network $t(s)$.

\section{The Sallen-Key Circuit Topology}

The basic Sallen-Key circuit configuration is shown in Fig. 1(a). The four-terminal network $t(s)$ is passive $R C$. An operational amplifier in the noninverting mode is used as a voltage amplifier with closed-loop gain $\beta$; $t(s)$ is characterized by the following voltage transfer parameters:

$$
\begin{aligned}
& t_{31}=\left.\frac{E_{3}}{E_{1}}\right|_{E_{2}=0}=\frac{n_{31}}{d_{31}} \\
& t_{32}=\left.\frac{E_{3}}{E_{2}}\right|_{E_{1}=0}=\frac{n_{32}}{d_{32}} .
\end{aligned}
$$

Since $d_{31}=d_{32}=d$, the overall transfer function is given by

$$
T(s)=\frac{E_{\text {out }}}{E_{\text {in }}}=\frac{\beta t_{31}}{1-\beta t_{32}}=\frac{n_{31} \cdot \beta}{d-\beta n_{32}} .
$$

Adding a path between $A-A^{\prime}$ and $B-B^{\prime}$ to the loaded twin-T shown in Fig. 2 and using this as the fourterminal $R C$ network $t(s)$, one obtains the circuit shown in Fig. 1(b). This configuration provides every possible second-order amplitude response merely by disconnecting appropriate components or interconnection paths
[8], [9]. This feature is necessary for HIC design where the common topology of Fig. 1(a) permits batch processing and handling, irrespective of the actual function required. Thus a single general-purpose filter building block containing all the components and conducting paths shown in Fig. 1(b) can be fabricated in large quantities. The component and conductor disconnections necessary to generate a particular network function can be subsequently carried out by some process that is compatible with HIC technology (e.g., scribing, etching, cutting, etc.).

A summary of all possible amplitude functions derived from the general circuit shown in Fig. 1(b), simply by deleting the appropriate components, is shown in Tables I-III. As in Fig. 1(b), all resistors $R_{i}$ and capacitors $C_{i}$ are referred to a given resistor $R$ and capacitor $C$ by a corresponding factor $r_{i}$ and $c_{i}$, respectively. The functions listed are special cases of the most general second-order function:

$$
T(s)=K \frac{s^{2}+\frac{\omega_{z}}{q_{z}} s+\omega_{z}{ }^{2}}{s^{2}+\frac{\omega_{p}}{q_{p}} s+\omega_{p}^{2}} .
$$

For the topology in Fig. 1, the undamped natural frequencies $\omega_{z}$ and $\omega_{p}$, as well as the zero- $Q q_{z}$, are functions of $R$ 's and $C$ 's only. Only the pole- $Q q_{p}$ depends on $\beta$, namely, by a function of the form

$$
q_{p}=\kappa_{1} /\left(\kappa_{2}-\kappa_{3} \beta\right)
$$

where the $\kappa_{i}$ are functions of $R$ 's and C's. Design equations for the coefficients corresponding to each particular network function are given in Tables I-III. $Q$-sensi- 
TABLE II

SECOND-ORDER S\&K NETWORKS

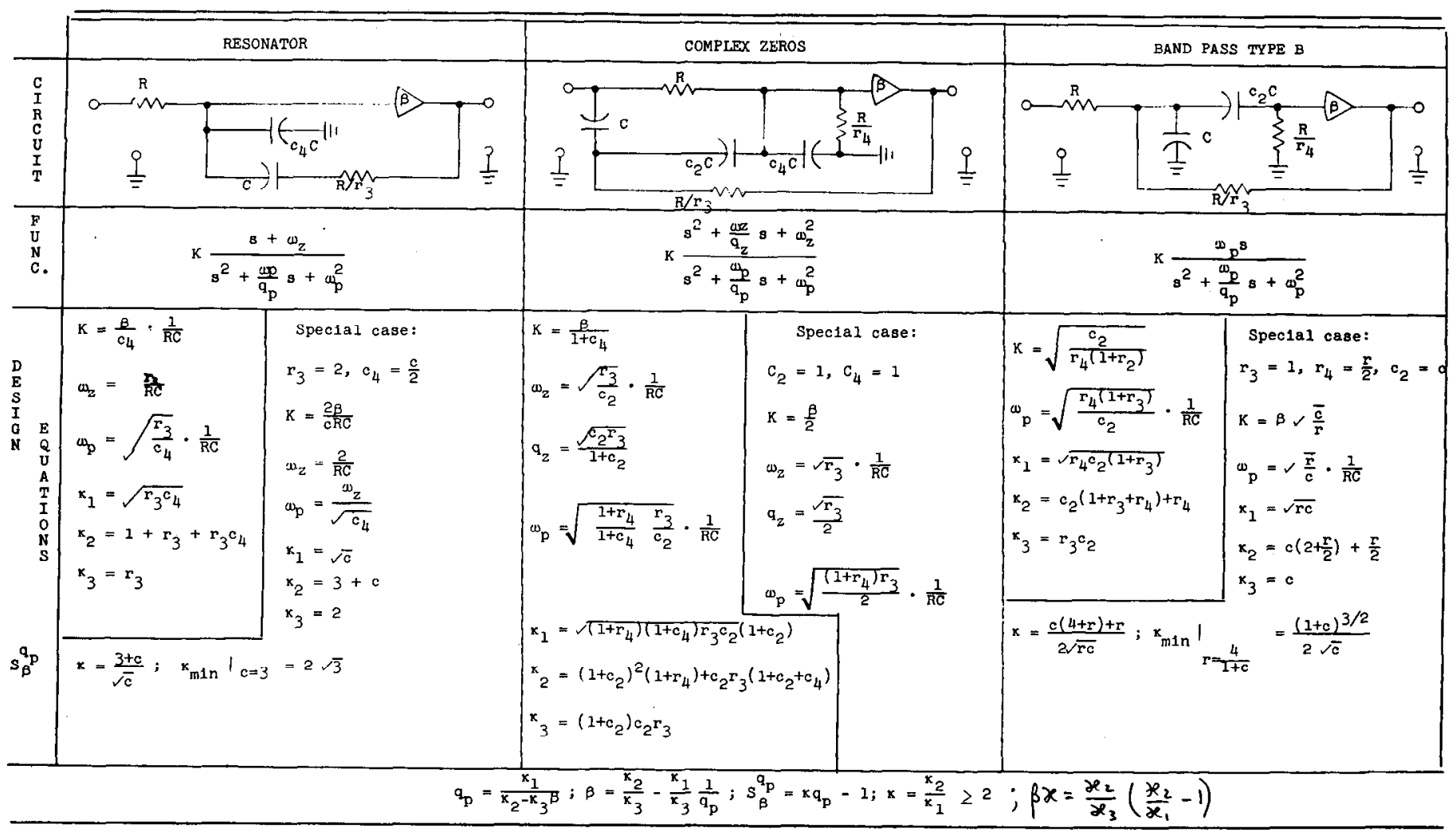

TABLE III

Second-Order S\&K Networks-Frequency Rejection NETWORKS (I.E., IMAGINARY ZEROS)

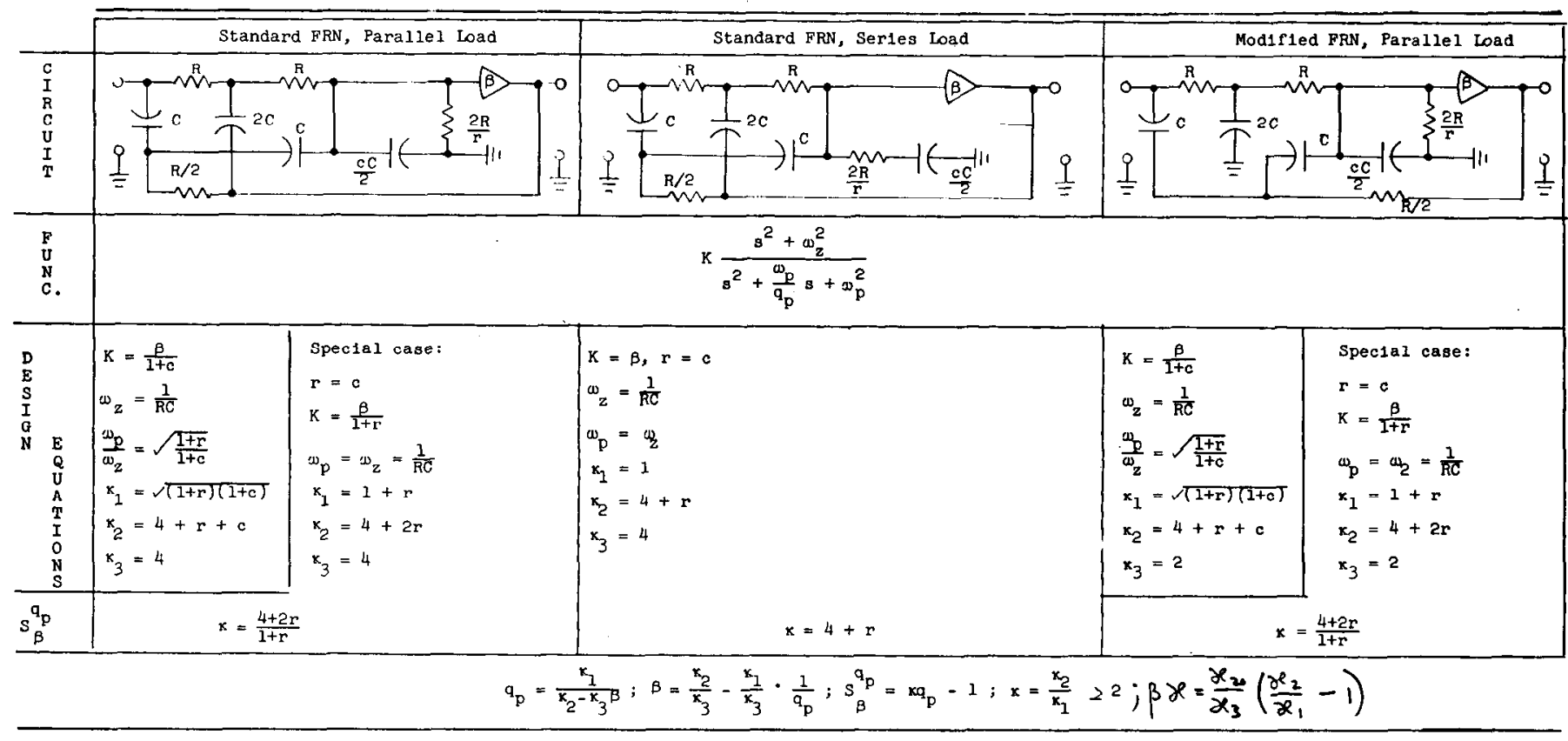

tivities to variations in the voltage gain $\beta$ are also given. These will be discussed in more detail in Section III.

It is of interest to note here that the pole- $Q$ of the passive $R C$ network $t(s)$, used in the basic circuit diagrams shown in Fig. 1 (a) and (b), results from (5) by setting $\beta$ equal to zero. Designating the pole- $Q$ of $t(s)$ by $q_{R}$, we obtain

$$
q_{R}=\left.q_{p}\right|_{\beta=0}=\frac{\kappa_{1}}{\kappa_{2}}<0.5
$$




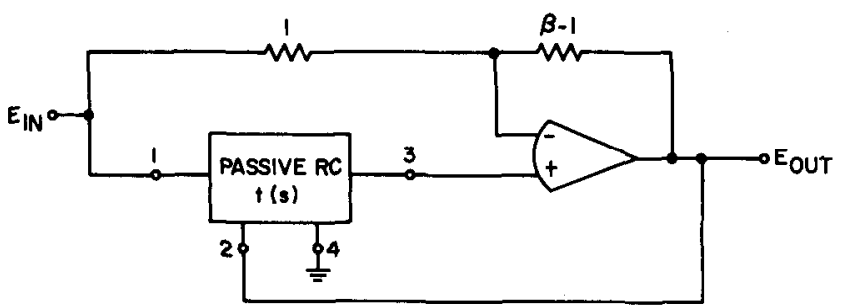

(a)

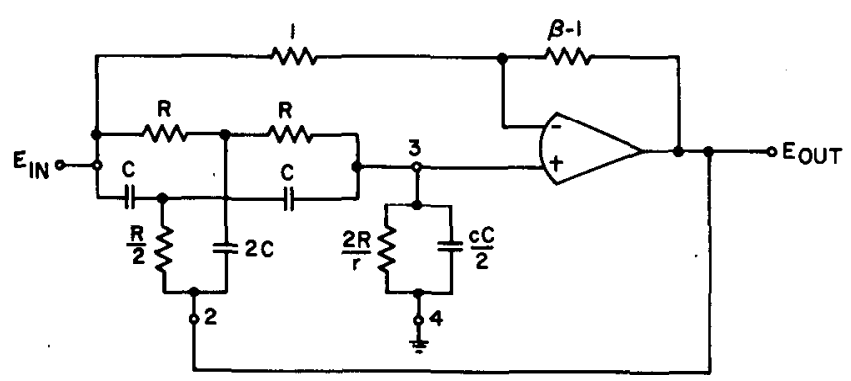

(b)

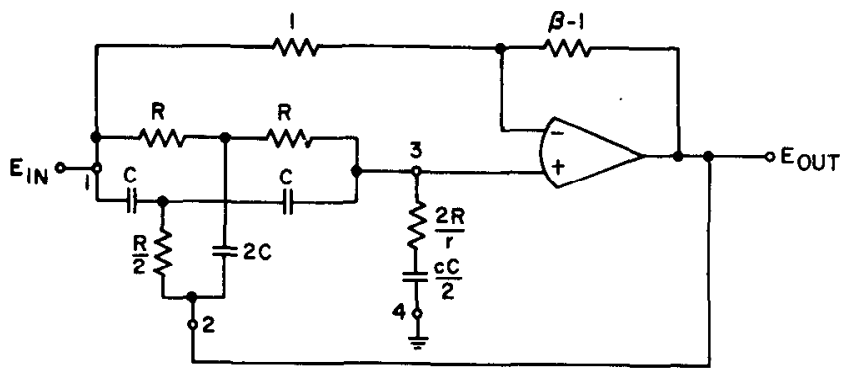

(c)

Fig. 3. All-pass network using SABB. (a) Basic topology. (b) Parallel-loaded twin- T. (c) Series-loaded twin-T.

The inequality in (6) sets a constraint on the $\kappa_{1}$ and $\kappa_{2}$ values given in Tables $\mathrm{I}-\mathrm{III}$.

Fortunately, a very simple modification of the basic configuration shown in Fig. 1(a) provides a secondorder all-pass network whose poles are realized in exactly the same way as those of the amplitude sections described in the preceding section. The all-pass configuration is shown in Fig. 3(a). Note that the only difference with respect to the configuration in Fig. 1(a) is that the ground connection of the $\beta$-feedback network has been connected to the input. Using a null network (e.g., twin-T or bridged-T) for $t(s)$, an all-pass network can be obtained [10]. Using an $R C$-loaded (series or parallel) twin- $T$, the circuits shown in Fig. 3(b) and (c) are obtained. Note that these configurations are contained in the general circuit of Fig. 1(b), as are bridgedT's, for use as $t(s)$. The latter is less complex and may be tuned more easily in many cases.

\section{Sensitivity Considerations}

Much has been written on the pros and cons of SallenKey $Q$-stability [11]-[13], and various alternatives claiming lower $Q$-sensitivity have been advanced.

In discussing the sensitivity of active networks here, the following assumptions will be made.
1) The main incentive for going to active filters is to utilize the economies of batch processing offered by IC technology. Therefore, IC implementation will be assumed exclusively.

2) To cope with the problem of active network sensitivity, the demands on passive component stability are high. Passive silicon integrated circuit (SIC) componcnts cannot meet these demands. Thin-film componerits meet them well. Hybrid IC implementation will therefore be assumed here, consisting of tantalum thinfilm $R C$ combinations [TIC's] and active SIC's.

3) TIC resistors track very closely on a substrate (better than $\pm 5 \mathrm{ppm} /{ }^{\circ} \mathrm{C}$ ) as do $\mathrm{TIC}$ capacitors $\left( \pm 10 \mathrm{ppm} /{ }^{\circ} \mathrm{C}\right)$. Temperature coefficients of resistance (TCR) and temperature coefficients of capacitance (TCC) can be matched closely. With the excellent aging stability of TIC resistors and capacitors, a worst-case frequency tolerance of $0.36-0.5$ percent can be assumed over a 5-10-year life and over a $40-60^{\circ} \mathrm{C}$ ambient temperature range, respectively.

Taking these assumptions into account, it can be shown [14] that the frequency and $Q$-variation of a hybrid-integrated network whose poles are determined by an equation of the form

$$
s^{2}+\frac{\omega}{Q} s+\omega^{2}=0
$$

are given by

$$
\frac{\Delta \omega}{\omega}=-\left(\frac{\Delta R}{R}+\frac{\Delta C}{C}\right)+S_{\beta} \omega \frac{\Delta \beta}{\beta}
$$

and

$$
\frac{\Delta Q}{Q}=S_{\beta} Q \frac{\Delta \beta}{\beta} .
$$

Considering frequency stability first, we find by inspection of Tables I-III that the pole frequency $\omega_{p}$ (designated $\omega$ here for brevity) is independent of $\beta$. Thus for Sallen-Key-type networks, (8) simplifies to

$$
\frac{\Delta \omega}{\omega}=-\left(\frac{\Delta R}{R}+\frac{\Delta C}{C}\right) .
$$

The frequency stability is therefore primarily dependent on the stability and temperature coefficient matching capabilities of the passive components. This, in turn, depends directly on the state of the component art which, as mentioned above, is presently of the order of $0.36-0.5$ percent depending on ambient conditions and service life. This compares favorably with the frequency stability obtainable with standard discrete $L C$ equivalents.

Addressing ourselves to the question of $Q$-variations [15] we have, from (9),

$$
\frac{\Delta Q}{Q}=V_{\beta}^{Q}=S_{\beta}^{Q} \cdot \frac{\Delta \beta}{\beta}
$$


where $V_{x} F$ is defined as the variation of the function $F$ resulting from changes in $x$. From (5) and (6) we have ${ }^{1}$

$$
S_{\beta}{ }^{Q}=\frac{\kappa_{2}}{\kappa_{1}} Q-1=\frac{Q}{q_{R}}-1=\kappa Q-1
$$

where $\kappa=\kappa_{2} / \kappa_{1}=1 / q_{R}$. From (6) it follows directly that

$$
\kappa>2 .
$$

This inequality has also been derived in the literature [12]. Clearly, to minimize the sensitivity $S_{\beta}{ }^{Q}$ for any given network function $T(s), \kappa$ must be minimized by an appropriate choice of resistors and capacitors. For the second-order circuits in Tables I-III, $\kappa$ is a function of three variables or ratios $r_{i}$ and $c_{i}$. A special case is given for some of the circuits in Tables I-III, where a value for one of the three component ratios has been assumed and $\kappa$ optimized with respect to one of the others. $\kappa$ is minimized with respect to the third by letting it either become very large or tend to zero. For the special cases shown in Tables I-III, $r$ and $c$ can readily be chosen such that $\kappa$ typically lies between 2 and 2.5, i.e., very close to the minimum value. Incidentally, sensitivity minimization is only one of the considerations that determines the choice of the component ratios. It may well be that for other considerations, such as minimum substrate area of thin-film components or maximum circuit versatility, it is worth accepting a certain deterioration in sensitivity.

Minimizing $S_{\beta}{ }^{Q}$ by minimizing $\kappa$ becomes much simpler when we recognize that $\kappa$ is the inverse pole- $Q$, namely $q_{R}$, of the passive $R C$ network $t(s)$ in Figs. 1(a) and $3(\mathrm{a})$. Thus minimizing $\kappa$ reverts to the problem of maximizing $q_{R}$. Since we are considering only secondorder networks, $q_{R}$ is related to the distance between the two poles of $t(s)$ on the negative-real axis. The maximum value of $q_{R}$ (i.e., 0.5 ) is attained when the two poles coincide' on a double pole. This condition can be approximated reasonably well by physically realizable networks; whereas a $k=1 / q_{R}$ value of 2 cannot be attained, a value of 2.2 does not strain the physical realizability of a network at all.

It can be shown that in order to maximize $q_{R}$, the two sections of the $R C$ network providing its two poles should be isolated from each other as much as possible. Rather than use a buffer amplifier between them, it is much more economical to achieve a certain amount of isolation by impedance mismatching. This can be obtained by starting out with two identical pole sections and impedance scaling one of them with respect to the other. Consider, for example, the ladder network shown in Fig. 4 and assume that a network $\mathrm{N}_{a}$ defines a pole $p_{1}$. The two poles realized by $\mathrm{N}_{a}$ in cascade with $\mathrm{N}_{b}$ will approach a double pole as $\rho \gg 1$. At the same time, of course, $\kappa$ will approach its minimum of 2 .

\footnotetext{
1 The $Q$ under consideration here is the pole- $Q$ designated $q_{p}$ in (5) and in the design equations of Tables I-III.
}

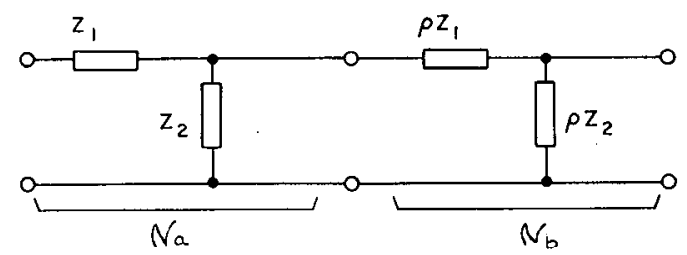

Fig. 4. Second-order ladder network; closely spaced pole pair achieved by impedance scaling.

To obtain $V_{\beta}{ }^{Q}$ we must now estimate the value of $\Delta \beta / \beta$, which is the relative change of the closed-loop gain $\beta$ of the noninverting operational amplifiers shown in Figs. 1 and 3. This has been dealt with in detail in [16], where it is shown that for the case that highly stable resistors (e.g., thin film) are used for the feedback network of the amplifier, the closed-loop gain $\beta$ is most critical to variations in open-loop gain $A$. We must therefore derive the following variation:

$$
\frac{\Delta \beta}{\beta} \approx V_{A^{\beta}}=S_{A}{ }^{\beta} \frac{\Delta A}{A} .
$$

In $[14]$ it is shown that

$$
S_{A}^{\beta}=\frac{1}{1+L G} \approx \frac{1}{L G}=\frac{\beta}{A}
$$

where $L G$ is the loop gain of the amplifier. Thus from (11) and (15),

$$
\frac{\Delta Q}{Q}=\left[\beta \cdot S_{\beta}{ }^{Q}\right] \cdot \frac{\Delta A}{A^{2}}=\Gamma \cdot \frac{\Delta A}{A^{2}}
$$

where

$$
\Gamma=\beta \cdot S_{\beta}^{Q}
$$

is called the gain-sensitivity product. It is a useful measure for the $Q$-stability of hybrid-integrated active filter networks using operational amplifiers.

Since the term $\Delta A / A^{2}$ in (16) depends on the operational amplifier, the circuit designer has virtually no control over its value. This leaves the term $\Gamma$, which must be minimized in order to minimize $\Delta Q / Q$. For the general Sallen-Key network, we can express $\Gamma$ in terms of the desired pole- $Q$; with the terms in (5), (6), and (12) it follows that

$$
\Gamma=\beta S_{\beta}^{Q}=(\beta \kappa)\left[Q-q_{R}\right]=\frac{\kappa_{2}}{\kappa_{3}} \frac{\left(Q-q_{R}\right)^{2}}{Q q_{R}} .
$$

The most direct way of minimizing $\Gamma$ is to minimize the $(\beta \kappa)$ product. For any given circuit, this can be done directly by computer. The circuit elements representing degrees of freedom are selected at random and the configuration with the minimum $\beta \kappa$ product found by a Monte Carlo routine. The resulting circuit will have minimum $Q$-variation with respect to changes in the active element (i.e., the amplifier $\beta$ ). It is interesting to note that the configuration for which the $\beta \kappa$ product is a minimum differs only negligibly from the configuration for which the $Q$-variation is minimum, with respect to 


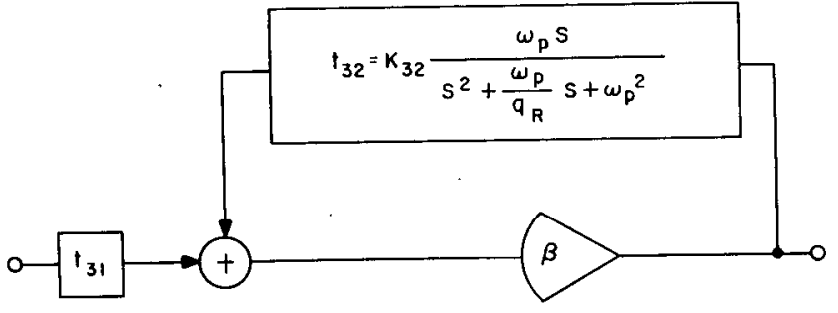

Fig. 5. Fig. 1(a) redrawn as feedback network with $R C$ bandpass filter in feedback path.

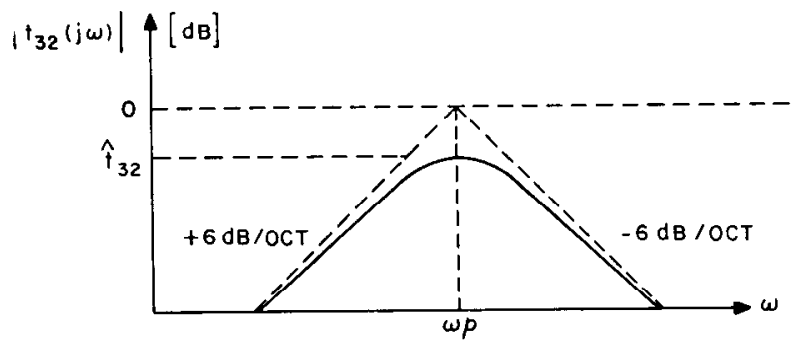

Fig. 6. Frequency response of feedback network $t_{82}$.

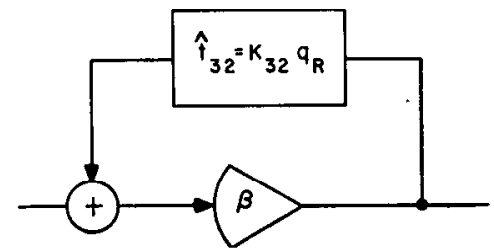

Fig. 7. Feedback configuration at pole frequency $\omega_{p}$.

the active and the thin-film passive components. Thus minimizing the $\beta \kappa$ product optimizes the HIC also if the small variations of the passive thin-film components are taken into account.

To obtain a lower bound on $\Gamma$ it is useful to look more closely at the ratio $\kappa_{2} / \kappa_{3}$. For this purpose we return to the defining transfer function (3) and find that the passive $R C$ transfer function $t_{32}(s)$ must have the form

$$
t_{32}=K_{32} \cdot \frac{\omega_{p} s}{s^{2}+\frac{\omega_{p}}{q_{R}} s+\omega_{p}^{2}} .
$$

This is the transfer function of a passive $R C$ secondorder bandpass network. We can now redraw the general block diagram of Fig. 1(a) in the equally general but more informative block diagram shown in Fig. 5 . From (19) it follows that the peak value $\hat{t}_{32}$, which occurs at $\omega_{p}$ (see Fig. 6), is given by

$$
\hat{t}_{32}=t_{32}\left(j \omega_{p}\right)=K_{32} \cdot q_{R} .
$$

Returning to the feedback loop in Fìg. 5 and considering it at the frequency $\omega_{p}$ we obtain the configuration shown in Fig. 7. For oscillation to occur (i.e., $Q \rightarrow \infty$ ) it follows by inspection that $\beta$ must take on the value

$$
\beta_{\mathrm{osc}}=\left(\hat{l}_{32}\right)^{-1}=\left(K_{32} q_{R}\right)^{-1} .
$$

However, from (5) it follows that the $\beta$ required for infinite $Q$ is given by

$$
\beta_{\text {osc }}=\kappa_{2} / \kappa_{3} .
$$

Combining (21) and (22),

$$
\kappa_{2} / \kappa_{3}=\left(\hat{t}_{32}\right)^{-1}=\left(K_{32} q_{R}\right)^{-1}
$$

and (18) becomes

$$
\Gamma=\beta S_{\beta}^{Q}=\frac{1}{\hat{t}_{32}} \frac{\left(Q-q_{R}\right)^{2}}{Q q_{R}}=\frac{1}{K_{32}} \frac{\left(Q-q_{R}\right)^{2}}{Q q_{R}^{2}} .
$$

For $Q \gg q_{R}$ this simplifies to

$$
\Gamma \approx \frac{1}{\hat{t}_{32}}\left(\frac{Q}{q_{R}}\right)=\frac{1}{K_{32}} \frac{Q}{q_{R}^{2}} .
$$

The problem of minimizing the gain-sensitivity product $\Gamma$ is now reduced to that of maximizing $\hat{t}_{32}$ or $q_{k}$ and $K_{32}$. Considering $\hat{t}_{32}$ first, it can be shown that the maximum transmission of a passive sccond-order $R C$ network is approximately unity $;^{2}$ therefore,

$$
0<\hat{t}_{32}=\left|t_{32}(j \omega)\right|_{\max }<1 .
$$

Thus from (6) and (24),

$$
\Gamma \geq\left[2 Q+\frac{1}{2 Q}-2\right]_{Q>>1} \approx 2(Q-1)
$$

A more accurate lower bound on $\Gamma$ can be obtained by considering the individual coefficients $K_{32}$ and $q_{R}$. We know the limits of $q_{R}$ to be between 0 and 0.5 .

Furthermore, since $t_{32}(s)$ is the transfer function of a 3-terminal $R C$ network, $K_{32}$ is limited by

$$
0<K_{32}<2+1 / q_{R}
$$

in order that $0<t_{32}(s)<1$ for $0<s<\infty$ [18]. With (24) we then have

$$
\Gamma=\frac{\left(Q-q_{R}\right)^{2}}{K_{32} Q q_{R}{ }^{2}} \geq \frac{\left(Q-q_{R}\right)^{2}}{\left(2+\frac{1}{q_{R}}\right) Q q_{R}{ }^{2}}=\frac{\left(Q-q_{R}\right)^{2}}{Q q_{R}\left(2 q_{R}+1\right)} .
$$

This expression decreases with increasing $q_{R}$. Thus for maximum $q_{R}$, namely $0.5,(28)$ implies that $\Gamma$ $\geq[Q-0.5]^{2} Q^{-1}$ or, for large $Q, \Gamma \geq Q-1$. This conclusion is true for those cases in which $t_{32}(s)$ is a general $R C$ network. However, for those cases in which $t_{32}(s)$ is an $R C$ ladder network, $K_{32}$ and $q_{R}$ are not independent of each other, as the following simple argument shows. Consider the two frequency responses corresponding to two different second-order bandpass functions $t_{32 A}$ and $t_{32 B}$ shown in Fig. 8. The corner frequencies $\omega_{p_{1}}$ and $\omega_{p_{2}}$ of our first case $l_{32 A}$ are far apart, corresponding to a low

${ }^{2}$ The maximum of a general second-order $R C$ network may actually exceed unity slightly [17], its upper bound being $\sqrt{4 / 3}$. For a ladder network the upper bound is unity. 


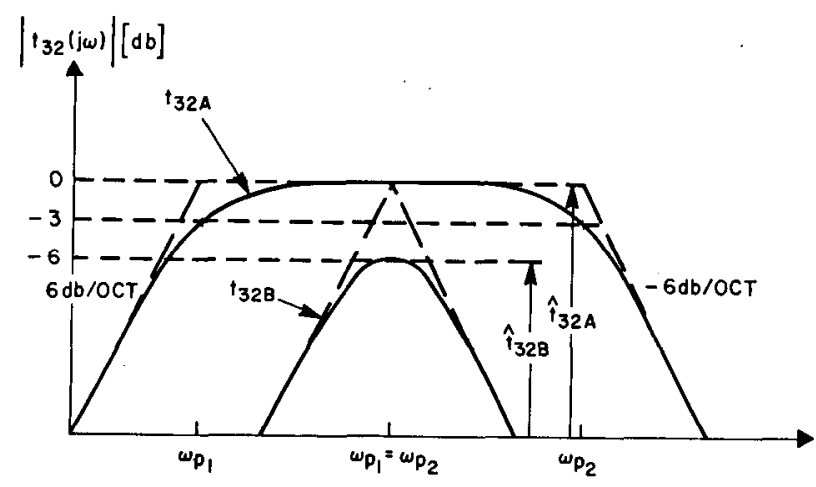

Fig. 8. Frequency response of $t_{32}(j \omega)$ for two $q_{R}$ values: $t_{32 A}$ corresponds to $\dot{q}_{R} \ll 0.5 ; t_{32 B}$ corresponds to $q_{R}=0.5$.

value of $q_{R}$; but permitting $t_{32 A}$ to reach its maximum value, $\hat{t}_{32 A}$ is equal to unity. By contrast, the corner frequencies of our second case $t_{32 B}$ coincide (i.e., $\omega_{p_{1}}=\omega_{p_{2}}$ ), corresponding to the maximum value of 0.5 for $q_{R}$ and, at the same time, to an attenuation of 0.5 (i.e., $-6 \mathrm{~dB})^{3}$ for $\hat{t}_{32 B}$. Thus we note that $q_{R}$ and $\hat{t}_{32}$ as well as $K_{32}$ cannot have maximum values simultaneously. Adding a prime to the maximum $\hat{l}_{32}$ and $K_{32}$ values compatible with maximum $q_{R}$, we have

$$
\hat{t}_{32}^{\prime}=\left.\hat{t}_{32}\right|_{q_{R}}=0.5 \leq 0.5 \text {. }
$$

Consequently, with (20),

$$
K_{32}{ }^{\prime}{ }_{\max }=\left.K_{32 \max }\right|_{q_{R}=0.5}=2 \cdot \hat{t}_{32}{ }^{\prime} \max =1 .
$$

From (24), the minimum gain-sensitivity product $\Gamma$ then results as

$$
\left.\Gamma\right|_{\kappa_{32-1}}=Q / q_{R}^{2}+Q^{-1}-2 / q_{R}=\kappa^{2} Q+Q^{-1}-2 \kappa
$$

where $\kappa=1 / q_{R}$. For $Q \gg 1$ we have

$$
\left.\Gamma\right|_{K_{32=1}} \approx \kappa^{2} Q-2 \kappa
$$

and with (6) the lower limit on $\Gamma$ results as

$$
\Gamma=\beta S_{\beta}^{Q} \geq 4(Q-1) \text {. }
$$

With (18), we have for the $\beta \kappa$ product (which is the ratio $\left.\beta / q_{R}\right)$

$$
\beta_{\kappa} \geq 4(Q-1) /\left(Q-q_{R}\right) \approx 4(Q-1) / Q .
$$

For $Q \gg 1$ the lower bound on this product is 4 . The lower bounds in (33) and (34) provide useful references with respect to which the gain-sensitivity product of any practical circuit can be measured.

With the expressions derived earlier, we have a simple and accurate method with which to design our circuits

${ }^{3}$ Assume that the bandpass network consists of a first-order lowand high-pass section, each with a pole at $\omega_{p}$. The minimum attenuation of each section at $\omega_{p}$ is $-3 \mathrm{~dB}$; the minimum attenuation of the two in cascade is therefore $-6 \mathrm{~dB}$.

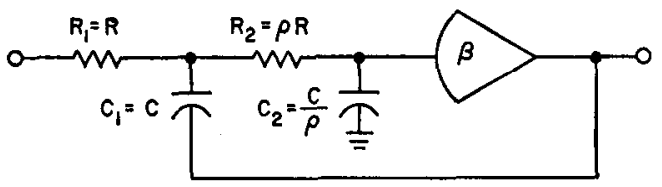

Fig. 9. Low-pass network with impedance-scaled feedback network.

for minimum gain-sensitivity product. In doing so it is preferable to calculate the $\beta_{\kappa}$-product rather than $\Gamma$, since the former is independent of $Q$. From (18) we have

$$
\beta_{\kappa}=\left.\frac{\Gamma}{Q-q_{R}}\right|_{Q \gg>q_{R}} \approx \frac{\Gamma}{Q} .
$$

Hence with (24),

$$
\beta_{\kappa}=\left.\frac{1}{K_{32} q_{R}^{2}}\left(1-\frac{q_{R}}{Q}\right)\right|_{Q>>q_{R}} \approx \frac{1}{K_{32} q_{R^{2}}^{2}} .
$$

Assuming that $Q \gg q_{R}$, the rightmost expression in (36) readily permits us to calculate and minimize $\beta \kappa$. For this purpose some typical forms of the feedback network $t_{32}$ are shown in Tables IV and V together with the corresponding $K_{32}$ and $q_{R}$ values.

Take, for example, the type- $B$ bandpass circuit shown in Fig. 9. From (36) we obtain

$$
\left(\beta_{K}\right)_{B P_{B}}=\left(1+\frac{R_{b}}{R_{a}}\right) \frac{\left(1+\frac{R_{2}}{R_{1}}+\frac{C_{1}}{C_{2}}\right)^{2}}{\sqrt{\frac{R_{2}}{R_{1}} \frac{C_{1}}{C_{2}}}} \frac{R_{1}}{R_{2}}
$$

where, referring to Table $\mathrm{V} R_{1}=R_{a} R_{b} /\left(R_{a}+R_{b}\right)$. Impedance scaling the second half of the ladder section with respect to the first by the factor $\rho$, we have $R_{1}=R$, $C_{1}=C, R_{2}=\rho R, C_{2}=C / \rho$, and (37) becomes

$$
(\beta)_{{ }_{K B P} B}=\left(1+\frac{R_{b}}{R_{a}}\right)\left(2+\frac{1}{\rho}\right)^{2} \text {. }
$$

A spread of $10: 1$ is quite reasonable with film resistors so that with $R_{b} / R_{a}=0.1$ and $\rho=10$ we obtain $\beta_{\kappa}=4.85$. Naturally, the wider the resistor spread given by $R_{b} / R_{a}$ and $\rho$, the closer to the lower bound given by (34) we can come.

With (36) we can also compare the inherent capabilities of two circuits providing the same function. Consider, for example, the type- $A$ and type- $B$ bandpass circuits shown in Tables IV and V. For the type- $A$ circuit we obtain

$$
\left(\beta_{\kappa}\right)_{B P_{A}}=\left(1+\frac{R_{b}}{R_{a}}\right) \frac{\left(1+\frac{R_{1}}{R_{2}}+\frac{C_{2}}{C_{1}}\right)^{2}}{\sqrt{\frac{R_{1}}{R_{2}} \frac{C_{2}}{C_{1}}}} .
$$


TABLE IV

Typical. Forms of the Feedback $R C$ Bandpass Network $t_{32}(s)$

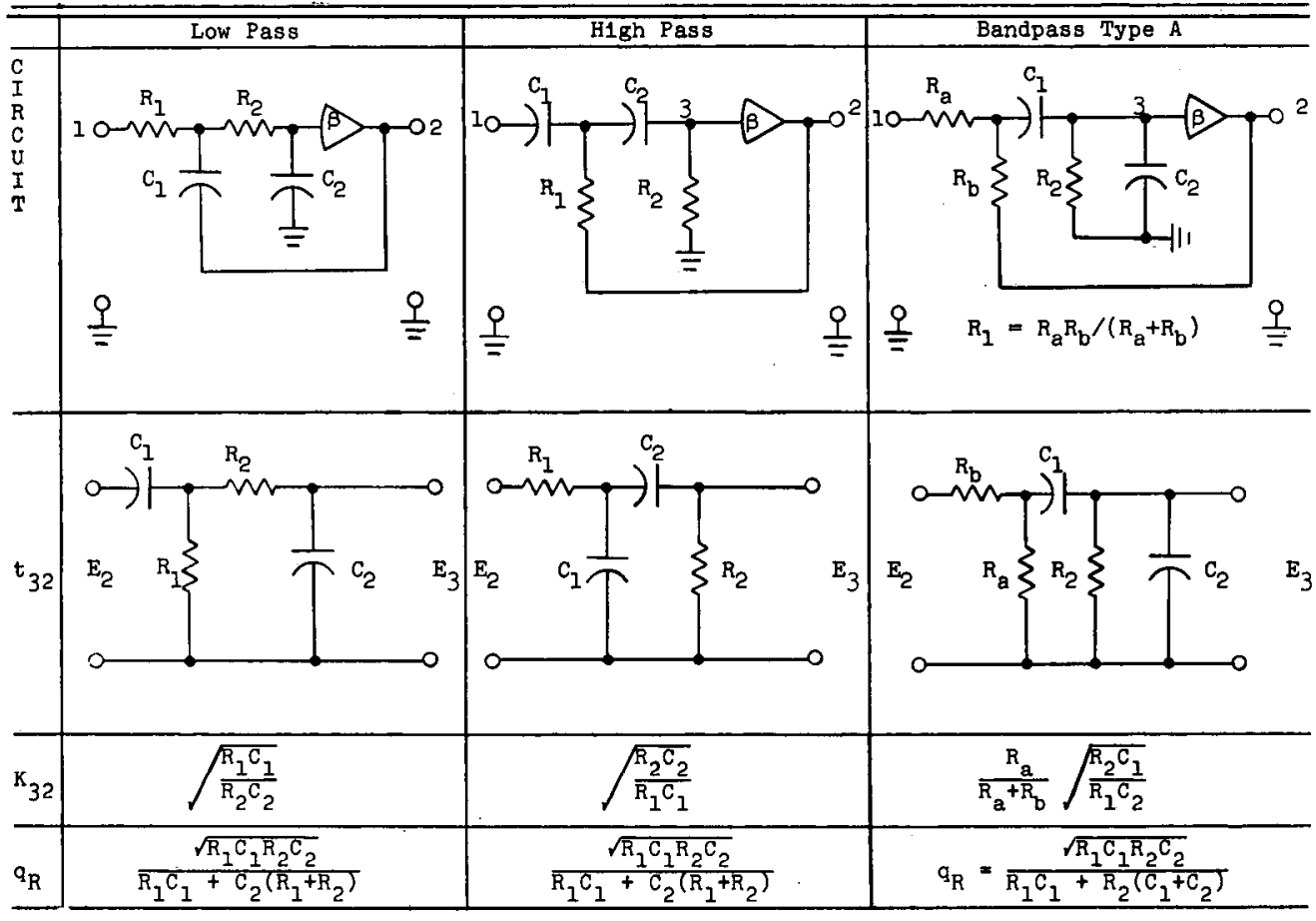

TABLE V

Typical Forms of the FeEdback $R C$ Bandpass Netwok $t_{32}(s)$

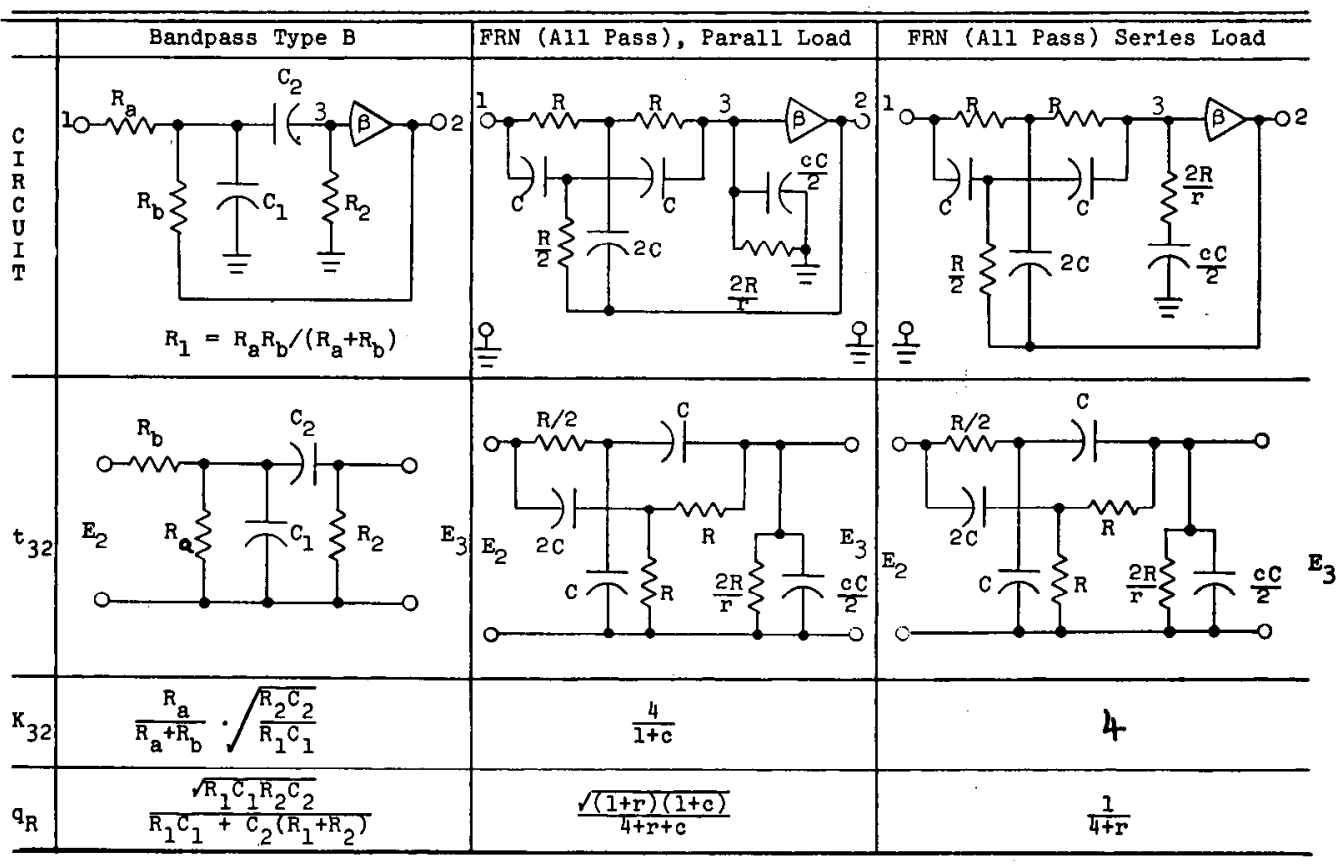

Comparing this with (38) the similarity in form is readily apparent. However, the additional multiplicand $R_{1} / R_{2}$ in (37) readily permits the minimum value of $(\beta \kappa)$ given by $(34)$ to be approached arbitrarily closely, by impedance scaling [see (38)]. This possibility does not exist for the type- $A$ circuit. Experimental results to this effect, showing that for a given $Q$ and $Q$-sensitivity, the $Q$-variation with temperature, say, is very much less for the type- $B$ than for the type- $A$ circuit, have been previously published in [14].

In general, a maximum pole- $Q$ variation $(\Delta Q / Q)_{\max }$ can be tolerated and a maximum open-loop gain varia- 
tion $(\Delta A / A)_{\max }$ is specified by the amplifier manufacturer. With (12) and (16) the maximum acceptable $Q$ of the Sallen-Key network is therefore limited to

$$
Q_{\max } \leq \frac{L G}{\kappa} \cdot\left[\frac{\Delta Q}{Q}\right]_{\max }\left[\frac{\Delta A}{A}\right]_{\max }^{-1}
$$

For example, if $[\Delta Q / Q]_{\max }=5$ percent, $[\Delta A / A]_{\max }=50$ percent, the available loop gain is 1000 , and $\kappa=2.5$, then the maximum acceptable $Q$ is 40 . Since the available loop gain is frequency dependent, i.e., it decreases with frequency, the maximum acceptable $Q$ will decrease with frequency as well. It is clear from (33) that it is worth designing the network such that the coefficient $\kappa$ remains reasonably close to its minimum value of 2 when the loop gain is small, such as when the frequency of operation is high.

\section{High- $Q$ Networks Using the \\ Sallen-Key Building Block}

The single-amplifier building block (SABB) described so far only covers relatively low- $Q$ applications in the order of 20 to 25 . For higher $Q$ values, FEN's must be used as discussed in detail in [2].

Experience has shown, however, that in many communications systems, only very few second-order sections with pole- $Q$ 's higher than 20 are required. In such cases, it is clearly not worth going to the expense of fabricating a hybrid-integrated building block incorporating the dual-amplifier FEN circuits. Instead, the SABB incorporating the Sallen-Key circuitry of Fig. 1 (b) can be used as the active twin- $T$ in the feedback loop of a FEN, capable of providing high- $Q$ poles as shown in Fig. 10(a). The additional forward amplifier $(\mu)$ and the two resistors $R_{F}$ and $R_{Q}$ may be external and discrete. It is important to remember that the active twin- $\mathrm{T}$ in the feedback loop of a FEN determines its frequency characteristics, and in particular its frequency stability. Since the active twin-T in the configuration of Fig. 10(a) is integrated, i.e., its stability is determined by the thin-film passive components, no sacrifice in frequency stability results. For the active input network to the FEN, an additional hybrid integrated SABB is used, as shown in Fig. 10(b). This approach is particularly economical, since it permits the use of both highand low- $Q$ filter networks in quantity, while requiring the hybrid integrated production of only SABB's.

\section{Computer-Aided Filter Design}

The high degree of standardization and the universality of the building blocks described here strongly suggest the potential for almost total filter design by computer. This potential was recognized early in the development of the building block. As a result various computer programs, covering certain aspects of filter design with the building blocks, have been continuously in use. Recently, these and the additional necessary pro-

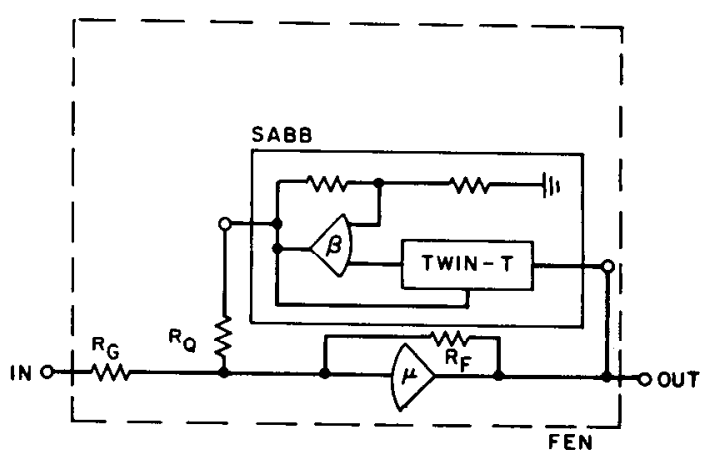

(a)

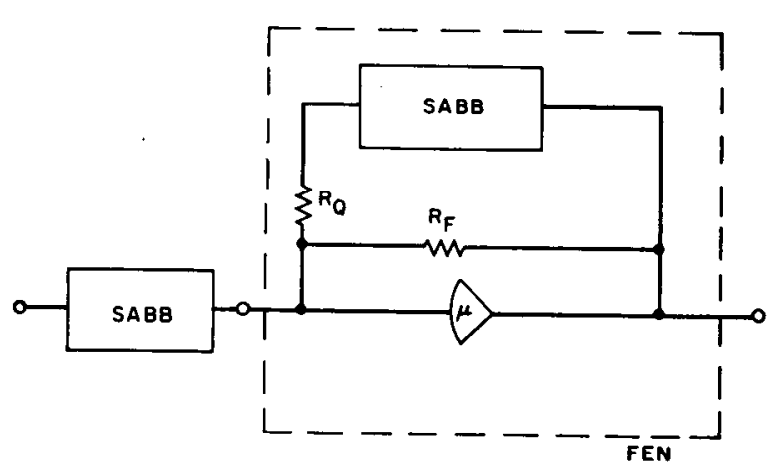

(b)

Fig. 10. (a) FEN using SABB in feedback loop. (b) General high- $Q$ second-order filter using $S A B B$ as input and feedback network.

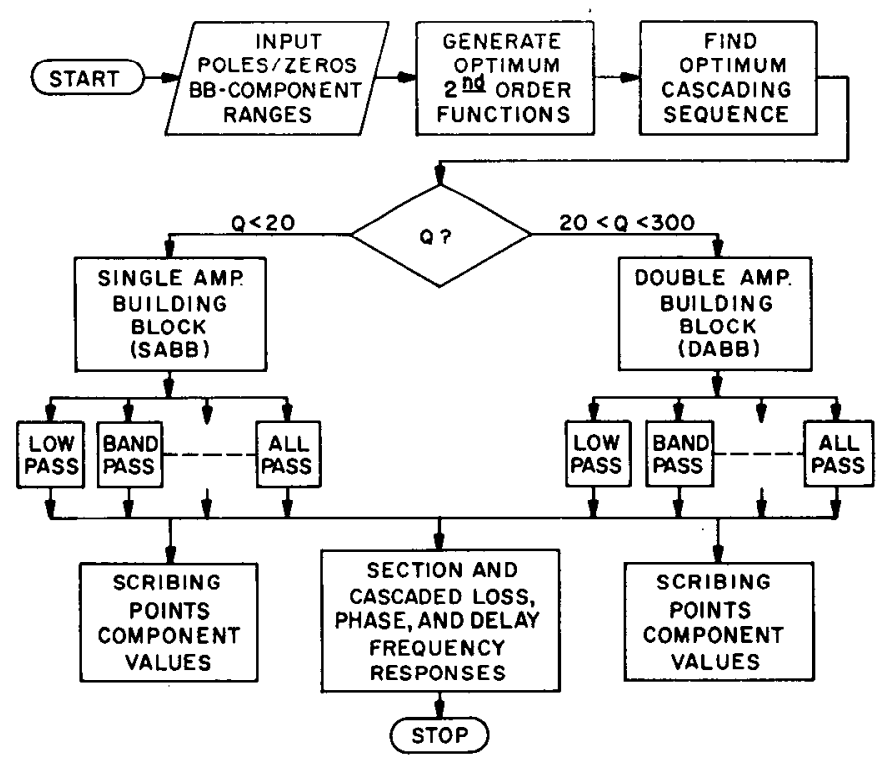

Fig. 11. Flow chart for computer-aided filter design.

grams have been compiled into one universal design program. The general flow chart [19] of this program is shown in Fig. 11. The program accepts the desired transfer function in terms of its polynomials or roots and pairs the resulting poles and zeros to minimize distortion, according to the Lueder-Halfin algorithm (see [4]). The pole-zero pairing determines the type of individual second-order functions, as well as their pole- $Q$. This in turn establishes whether the SABB or the 
double-amplifier building block (DABB), the latter incorporating a FEN, must be used. As output dala, the program provides the value of each component required for the particular building-block function in question, as well as the gain and phase response corresponding to this function. The program also prescribes the optimum sequence in which to cascade the final filter and provides the gain and phase response consecutively at the output of each section in this cascade; the output of the last stage being the desired filter response.

\section{Summary}

A highly versatile low- $Q$ active $R C$ filter building block is described. The building block is directly compatible with HIC technology in which thin-film resistors and capacitors are combined with a silicon monolithic operational amplifier chip. The circuit approach is also directly compatible with the FEN approach to high- $Q$ filter design; in fact, a simple method is shown of designing high- $Q$ frequency-stable FEN building blocks using the hybrid-integrated SABB and an external operational amplifier. In this way, a maximum number of SABB's and a minimum amount of development are guaranteed in production.

Design equations and design tables are given for any desired second-order function-any of which is realizable with the filter building block described, including nonminimum phase networks. Frequency and $Q$-sensitivity are examined in detail. Methods of minimizing $Q$-variation with respect to the active and passive elements are discussed, and lower bounds on the $Q$ variation obtainable with the hybrid-integrated filter building blocks are given.

\section{REFERENCES}

[1] R. P. Sallen and E. L. Key, "A practical method of designing RC active filters," IRE Trans. Circuit Theory, vol. CT-2, pp. 74-85, Mar. 1955.

[2] G. S. Moschytz, "FEN filter design using tantalum and silicon integrated circuits," Proc. IEEE, vol. 58, pp. 550-566, Apr. 1970 .

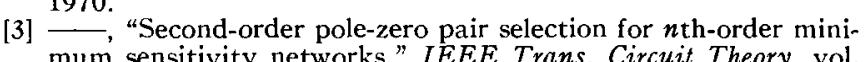
CT-17, pp. 527-534, Nov, 1970.

[4] E. Lueder, "A decomposition of a transfer function minimizing distortion and inband losses," Bell Syst. Tech. J., vol. 49, no. 3, pp. 455-469, Mar. 1970.

[5] W. II. Orr, R. M. Rickert, and D. M. Hill, "An integrated RC oscillator for TOUCH-TONE ${ }^{\odot}$ dialing," IEEE Trans. Commun. Technol., vol. COM-16, pp. 624-629, Aug. 1968.

[6] F. H. Hintzman, Jr., "Computer tuning of hybrid audio oscillators," IEEE Spectrum, pp. 56-60, Feb. 1969.

[7] W. H. Orr, "The computer design and precision tuning of thin film filters," in 1966 Seminar Integrated Circuits (Feb. 2, 1966), pp. 45-56, 1966.

[8] G. S. Moschytz, "Sallen and Key filter networks with amplifier gain larger than or equal to unity," IEEE J. Solid-State Circuits (Corresp.), vol. SC-2, pp. 114-116, Sept. 1967.

[9] G. S. Moschytz and W. Thelen, "The design of hybrid integrated-filter building blocks," IFEF J. Solid-State Circuits, vol. SC-5, pp. 99-107, June 1970

[10] G. S. Moschytz, "A general all-pass network based on the Sallen-Key circuit," IEEE Trans. Circuit Theory (Corresp.), vol. CT-19, pp. 392-394, July 1972.

[11] R. C. Foss and B. J. Green, " $Q$ factor, $Q$ stability and gain in active filters," Electron. Lett., vol. 2, no. 3, pp. 99-100, Mar. 1966.

[12] G. C. Bown, "Sensitivity in active RC filters," Electron. Lett., vol. 3, no. 3, pp. 298-299, Mar. 1967.

[13] W. Saraga, "Sensitivity of 2nd-order Sallen-Key-type active RC filters," Electron. Lett., vol. 3, no. 10, Oct. 1967.

[14] G. S. Moschytz, "Gain-sensitivity product-A figure of merit for hybrid-integrated filters using single operational amplifiers," IEEE J. Solid-Siate Circuits, vol. SC-6, pp. 103-110, June 1971 .

[15] 2 "A note on pole, frequency, and $Q$ sensitivity." IEEE $J$. Solid-State Circuits (Corresp.), vol.SC-6, pp. 267-269, Aug. 1971.

[16] - "The operational amplifier in linear active networks," IEEE Spectrum, vol. 7, pp. 42-50, Jan. 1970.

[17] A. D. Fialkow and I. Gerst, "The maximum gain of an RC network," Proc. IRE, vol. 41, pp. 392-395, Mar. 1953.

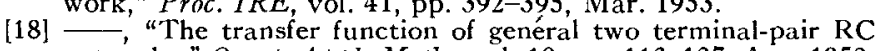
networks," Quart. A ppl. Math., vol. 10, pp. 113-127, Apr. 1952.

[19] . R. M. Ziegler, unpublished memorandum. 